\title{
Studies of the Polarimetric Covariance Matrix. Part II: Modeling and Polarization Errors
}

\author{
J. C. HubBerT* AND V. N. BRingi \\ Colorado State University, Fort Collins, Colorado \\ (Manuscript received 4 March 2002, in final form 2 January 2003)
}

\section{ABSTRACT}

\begin{abstract}
A polarimetric radar covariance matrix model is described to study the behavior of the co-to-cross covariances in precipitation. The $2 \times 2$ propagation matrix with attenuation, differential attenuation, and differential phase is coupled to the backscatter matrix leading to a propagation-modified covariance matrix model. System polarization errors are included in this model as well. This model is used to study the behavior of the magnitude and phase of the co-to-cross covariances and the linear depolarization ratio (LDR) in rainfall. It is shown that the model predictions are consistent with data collected with the Colorado State University (CSU)-University of Chicago-Illinois State Water Survey (CHILL) radar in intense rainfall. A method is also given for estimating the system polarization errors from covariance matrix data collected in intense rainfall.
\end{abstract}

\section{Introduction}

The $3 \times 3$ radar polarimetric covariance matrix, consisting of three real powers and three complex covariance elements, provides a complete set of measurements from distributed particles such as precipitation. It is only recently that research radars have been configured to measure all of the covariance matrix elements in real time (e.g., Brunkow et al. 2000). While the theory of covariance matrices and associated optimum polarizations for random media have been known for some time (e.g., Mott 1992, and extensive references therein), the application to retrieval of microphysical information of precipitation is much more recent (Huang et al. 2001; Ryzhkov et al. 2002). The conventional polarimetric observables such as the differential reflectivity $\left(Z_{\mathrm{dr}}\right)$, linear depolarization ratio (LDR), specific differential propagation phase $\left(K_{\mathrm{dp}}\right)$; and copolar correlation coefficient $\left(\rho_{\mathrm{HV}}\right)$ have been well studied in the past two decades with respect to their microphysical information content and are now, more or less, routinely applied to rain-rate estimation and hydrometeor-type classification (e.g., see Bringi and Chandrasekar 2001, and extensive references therein). The two co-to-cross covariance terms associated with $\left\langle S_{\mathrm{HH}} S_{\mathrm{VH}}^{*}\right\rangle$ and $\left\langle S_{\mathrm{VV}} S_{\mathrm{HV}}^{*}\right\rangle$ have only recently been receiving attention for the detection of

* Current affiliation: National Center for Atmospheric Research, Boulder, Colorado.

Corresponding author address: Dr. J. C. Hubbert, UCAR/ATD, Foothills Laboratory, Building 1, Room 2085, Boulder, CO 80303. E-mail: hubbert@ucar.edu oriented hydrometeors (Ryzhkov 2001; Ryzhkov et al. 2002), while the use of optimum polarizations has also only recently been investigated (Hubbert and Bringi 1996, 2001). There is promise that full covariance matrix analysis may lead to improved rain-rate estimation and to reducing ambiguities in hydrometeor classification based on the conventional five polarimetric measurands $\left(Z_{\mathrm{H}}, Z_{\mathrm{dr}}, \mathrm{LDR}, K_{\mathrm{dp}}, \rho_{\mathrm{HV}}\right)$. An important related application is experimental evaluation of the so-called "hybrid" mode proposed for polarimetric upgrade for the Weather Surveillance Radar-1988 Doppler (WSR88D) radars (Doviak et al. 2000). Clearly, more understanding of the behavior of the magnitude and phase of the co-to-cross covariances is needed, especially in the presence of system polarization errors. Also, techniques need to be developed for constructing accurate covariance matrices from data for which the system differential gain and phase offsets need to be evaluated. This paper addresses these issues using a covariance matrix modeling methodology and analysis of covariance matrix data from the Colorado State University (CSU)University of Chicago-Illinois State Water Survey (CHILL) radar. More specifically, the impact of the nonzero mean canting angle, spread of canting angles, and system polarization errors on the co-to-cross covariances and LDR are studied.

\section{Background}

As mentioned earlier, the members of the radar covariance matrix that result from the products of the copolar time series have been well studied (Doviak and Zrnić 1993; Bringi and Chandrasekar 2001). Because 


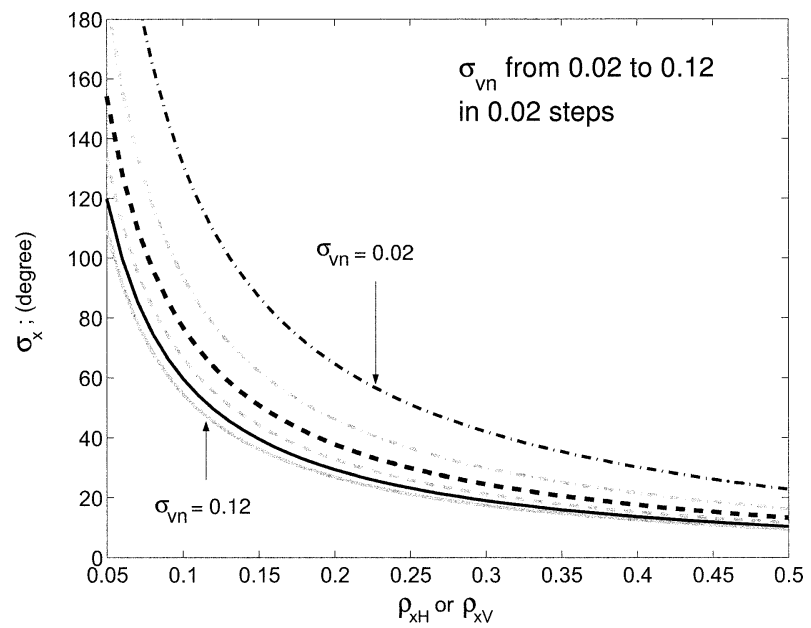

FIG. 1. The std dev of the phase of the co-to-cross covariance $\left(\phi_{\mathrm{xH}}\right.$ or $\left.\phi_{\mathrm{xV}}\right)$ as a function of the co-to-cross correlation coef $\left(\rho_{\mathrm{xH}}\right.$ or $\left.\rho_{\mathrm{xV}}\right)$ with the normalized spectral with $\sigma_{v n}$ as a parameter; $\sigma_{v n}$ $=\sigma_{\mathrm{v}} / \lambda /\left(2 T_{s}\right)$

the horizontal $(\mathrm{H})$ and vertical $(\mathrm{V})$ copolar signals are typically well correlated [if the signal-to-noise ratio (SNR) is at least $10 \mathrm{~dB}$ ], the data quality is high and the standard errors are low when at least 64 sample pairs are used per resolution volume (Liu et al. 1994). Recently, the calibration and interpretation of the co-tocross covariances has been a topic of interest (Hubbert et al. 1999; Hubbert and Bringi 2001; Ryzhkov 2001; Ryzhkov et al. 2002; Hubbert et al. 2003, hereafter Part I). The co-to-cross covariances are difficult to interpret not only because they result from the weaker cross polar time series but more importantly, because the cross polar time series is typically weakly correlated with the copolar time series, which results in high standard errors in the estimates, or "noisy" estimates. Figure 1 shows $\sigma_{x}$, the standard deviation of $\Psi_{\mathrm{xH}}$ (argument of the coto-cross covariance), as a function of $\rho_{\mathrm{xH}}$ (co-to-cross correlation coefficient for the $\mathrm{H}$ transmitted wave) with the normalized spectral width, $\sigma_{v n}$, as a parameter where $\sigma_{v n}=\sigma_{v} /\left(\lambda / 2 T_{s}\right)$, where $\sigma_{v}$ is the spectral width, $\lambda$ is the wavelength, and $T_{s}$ is the pulse repetition time. The Doppler spectra are assumed to be Gaussian shaped. The simulation is described by Chandrasekar et al. (1986) and Bringi and Chandrasekar (2001). As can be seen, for typical values of $\rho_{\mathrm{xH}}$ between 0.1 and 0.3 (e.g., in rain), the $\sigma_{x}$ varies from about $18^{\circ}$ to $140^{\circ}$ for spectral widths of $1-6 \mathrm{~m} \mathrm{~s}^{-1}$ (for $\lambda=10 \mathrm{~cm}$ and $T_{s}=1 \mathrm{~m} \mathrm{~s}$ ). Compare this to the standard deviation of $\phi_{\mathrm{dp}}$ (copolar differential propagation phase) at $\mathrm{S}$ band of about $2^{\circ}-$ $3^{\circ}$ (Sachidananda and Zrnić 1986).

It is known that the co-to-cross covariances are strong functions of mean canting angle and standard deviation of the canting angle of the precipitation medium. Ryzhkov (2001) has used an analytical model to study the effects of canting angle distribution on the co-to-cross covariances and has formulas for estimating the mean canting angle and standard deviation of canting angles of a rain medium. The interpretation of the co-to-cross covariances is perhaps clearer in the circular polarization basis. In terms of the circular polarization observables, the $\left\langle S_{\mathrm{HH}} S_{\mathrm{VH}}^{*}\right\rangle$ and $\left\langle S_{\mathrm{VV}} S_{\mathrm{HV}}^{*}\right\rangle$ elements can be expressed as

$$
\begin{aligned}
& \left\langle S_{\mathrm{HH}} S_{\mathrm{VH}}^{*}\right\rangle=\left[\rho_{2}\left\langle\eta_{c} \nu^{*}\right\rangle \sin 2 \beta_{0}+\rho_{4} \frac{\left\langle\eta_{c}|\nu|^{2}\right\rangle}{2} \sin 4 \beta_{0}\right], \\
& \left\langle S_{\mathrm{VV}} S_{\mathrm{HV}}^{*}\right\rangle=\left[\rho_{2}\left\langle\eta_{c} \nu^{*}\right\rangle \sin 2 \beta_{0}-\rho_{4} \frac{\left\langle\eta_{c}|\nu|^{2}\right\rangle}{2} \sin 4 \beta_{0}\right],
\end{aligned}
$$

where $\beta_{0}$ is the mean canting angle, $\rho_{2,4}$ are orientation parameters $\left(\rho_{2}=\langle\cos 2 \beta\rangle, \rho_{4}=\langle\cos 4 \beta\rangle\right)$ where angle brackets are expectations over a symmetric canting angle distribution about the mean $\beta_{0}, \nu$ is the circular backscatter amplitude ratio, $|\nu|^{2}$ is related to the circular depolarization ratio (CDR), and $\eta_{c}$ is the circular reflectivity (see Bringi and Chandrasekar 2001, section 3.5.3). For narrow Gaussian canting angle distributions, $\rho_{4}=\rho_{2}^{4}=\exp \left(-8 \sigma_{\beta}^{2}\right)$, where $\sigma_{\beta}^{2}$ is the variance of $\beta$ (Hendry et al. 1987). Rayleigh scattering is assumed. It follows that if $\beta_{0} \approx 0^{\circ}$, then the magnitude of the coto-cross covariances is nearly zero and the phase fluctuates between $0^{\circ}$ and $180^{\circ}$, depending on the sign of $\beta_{0}$. In practice, however, the mean canting angles are never identically zero and, in addition, small antenna polarization errors exist, both of which prevent the coto-cross covariances from being zero. In order to investigate these effects, a complete scattering model is developed here for the simulation of radar observables. Both the particles in the backscatter volume and the coherent propagation medium are independently modeled. The "steady" propagation medium is modeled via a $2 \times 2$ matrix that includes absolute attenuation $\left(A_{\mathrm{H}}\right)$, differential attenuation $\left(A_{\mathrm{dp}}\right)$, differential propagation phase $\left(\phi_{\mathrm{dp}}\right)$, and mean canting angle $(\theta)$ as parameters. The resolution volume (or backscatter medium) is modeled as an ensemble of precipitation particles with gamma drop size distribution (DSD) and various spatial orientation distributions via the T-matrix method (Vivekanandan et al. 1991; Waterman 1971). The modeled parameters can be independently varied so that the sensitivity of the cross polar and co-to-cross covariances can be studied. Antenna polarization errors are modeled similar to McCormick (1981).

\section{The scattering model}

The scattering geometry used here is the backscatter alignment (BSA) convention. The model strictly addresses the case of monostatic backscatter for precipitation particles that are rotationally symmetric. Canting angles are measured counterclockwise from the horizontal in the plane of polarization (i.e., plane containing $\mathrm{H}$ and $\mathrm{V}$ axes perpendicular to the propagation direction).

Because forward scatter is coherent (van de Hulst 
1957), the propagation medium can be completely described via a $2 \times 2$ scattering matrix $\mathbf{P}$ as

$$
\mathbf{P}=\mathbf{R}(-\theta) \mathbf{P}_{0} \mathbf{R}(\theta),
$$

where $\mathbf{R}$ is the Cartesian rotation matrix and $\mathbf{P}_{0}$ is the principal plane propagation matrix

$$
\mathbf{P}_{0}=\left[\begin{array}{cc}
e^{\left\{\lambda_{1} z\right\}} & 0 \\
0 & e^{\left\{\lambda_{2} z\right\}}
\end{array}\right],
$$

where $\lambda_{1,2}$ are the propagation constants along the principal planes of the propagation medium and $z$ is the distance along the direction of propagation. It follows that specific differential attenuation is $A_{\mathrm{dp}}=-(8.686$ $\left.\times 10^{3}\right) \Re\left\{\lambda_{1}-\lambda_{2}\right\} \mathrm{dB} \mathrm{km}{ }^{-1}$, and specific differential phase is $K_{\mathrm{dp}}=-10^{3} \mathfrak{\Im}\left\{\lambda_{1}-\lambda_{2}\right\} \mathrm{rad} \mathrm{km}^{-1}$. In rain these two parameters can be related by $A_{\mathrm{dp}}^{P}=\kappa K_{\mathrm{dp}}^{P}$ (Holt 1988; Bringi et al. 1990). The superscript $P$ signifies principal plane values. The characteristics of the propagation medium are then controlled via the variables $\theta$ (the mean canting angle) and $\kappa$. The output of the model gives radar measurands as a function of $\phi_{\mathrm{dp}}^{P}=2 \int_{r_{1}}^{r_{2}} K_{\mathrm{dp}}^{P} d r$.

The propagation medium is coupled to the backscatter medium via the radar-scattering matrix (Kennaugh 1949-1954; Sinclair 1950)

$$
\mathbf{S}=\left[\begin{array}{ll}
S_{a a} & S_{a b} \\
S_{a b} & S_{b b}
\end{array}\right]=\mathbf{P}^{\mathrm{T}}\left[\begin{array}{ll}
S_{\mathrm{HH}} & S_{\mathrm{HV}} \\
S_{\mathrm{HV}} & S_{\mathrm{VV}}
\end{array}\right] \mathbf{P},
$$

where $S_{\mathrm{HH}}, S_{\mathrm{VV}}$, and $S_{\mathrm{HV}}$ are backscatter amplitudes in the $\mathrm{H} / \mathrm{V}$ polarization basis. The propagation-modified covariance matrix is then formed as (Tragl 1990)

$$
\boldsymbol{\Sigma}_{0}=\left[\begin{array}{ccc}
\left\langle\left|S_{a a}\right|^{2}\right\rangle & \sqrt{2}\left\langle S_{a a} S_{a b}^{*}\right\rangle & \left\langle S_{a a} S_{b b}^{*}\right\rangle \\
\sqrt{2}\left\langle S_{a b} S_{a a}^{*}\right\rangle & 2\left\langle\left|S_{a b}\right|^{2}\right\rangle & \sqrt{2}\left\langle S_{a b} S_{b b}^{*}\right\rangle \\
\left\langle S_{b b} S_{a a}^{*}\right\rangle & \sqrt{2}\left\langle S_{b b} S_{a b}^{*}\right\rangle & \left\langle\left|S_{b b}\right|^{2}\right\rangle
\end{array}\right],
$$

where $\langle *\rangle$ denote ensemble average. Note that ensemble averaging only applies to the particles in the resolution volume (i.e., backscatter medium). Each matrix member in (6) consists of propagation terms and backscatter covariances of the form $\left\langle S_{x_{1} y_{1}} S_{x_{2} y_{2}}^{*}\right\rangle,\left(x_{1,2}, y_{1,2}=\mathrm{H}, \mathrm{V}\right)$. The $\mathrm{H} / \mathrm{V}$ basis backscatter covariance matrix $\boldsymbol{\Sigma}_{\mathbf{s}}$ is simulated via the T-matrix method for precipitation particles with specified size distribution (gamma), and orientation distribution (Fisher, Gaussian, etc.) with a mean canting angle of zero and some specified standard deviation of canting angle $\sigma_{\alpha}$. An arbitrary mean canting angle $(\alpha)$ and mean ellipticity angle can be given to the backscatter covariance matrix, $\boldsymbol{\Sigma}_{\mathbf{s}}$, by rotating this simulated covariance matrix in the plane of polarization with (Tragl 1990)

$$
\boldsymbol{\Sigma}_{\mathrm{HV}}(\chi)=\mathbf{T}^{-1}(\chi) \mathbf{\Sigma}_{\mathbf{s}} \mathbf{T}(\chi)
$$

where

$$
\mathbf{T}(\chi)=\Gamma^{2}\left[\begin{array}{ccc}
\rho^{2} & \rho^{2} \chi \sqrt{2} & \rho^{2} \chi^{2} \\
-\chi^{*} \sqrt{2} & 2\left(1-\chi \chi^{*}\right) & \sqrt{2} \chi \\
\rho^{* 2} \chi^{* 2} & -\rho^{* 2} \chi^{* 2} \sqrt{2} & \rho^{* 2}
\end{array}\right],
$$

where $\chi$ is the polarization ratio $\overline{\mathbf{E}}_{\mathrm{V}} / \overline{\mathbf{E}}_{\mathrm{H}}$, in which $\overline{\mathbf{E}}_{\mathrm{V}}$ and $\overline{\mathbf{E}}_{\mathrm{H}}$ are the electric field phasors, "*" signifies complex conjugation, and $\Gamma=\left(1+\chi \chi^{*}\right)^{0.5}$. As shown in Hubbert (1994), the phase term $\rho=e^{-j \tan ^{-1}(\tan \alpha \tan \epsilon)}$ is necessary to maintain a constant phase difference between the elliptic basis polarization vectors. The $\chi$ can be expressed in terms of the mean tilt (or canting) angle $\alpha$, and mean ellipticity angle $\epsilon$, as (Azzam and Bashara 1989)

$$
\chi=\frac{\tan \alpha+j \tan \epsilon}{1-j \tan \alpha \tan \epsilon} .
$$

To our knowledge, there are no known precipitation particles that yield a nonzero ellipticity angle in the $\mathrm{H} / \mathrm{V}$ basis and therefore $\epsilon$ is always taken as zero in this paper. This then completes the model. Summarizing, the backscatter medium is modeled via a covariance matrix $\left(\boldsymbol{\Sigma}_{\mathbf{s}}\right)$, which is calculated for a particular hydrometeor size distribution (gamma) and orientation distribution (Fisher, Gaussian, random, etc.) of precipitation particles with a zero mean canting angle $(\alpha)$ and specified spread of canting angles $\left(\sigma_{\alpha}\right)$. This ensemble of particles can be given an arbitrary mean canting angle via (7). The backscatter covariances are then substituted into (6); recall that each member of (6) consists of propagation terms and backscatter covariances. The parameters of the propagation matrix are controlled in (6) via (3). The mean canting angle of the propagation matrix $\boldsymbol{\theta}$ is set in (3) while the $A_{\mathrm{dp}}, A_{\mathrm{H}}$, and $\phi_{\mathrm{dp}}^{P}$ are specified in (4) via $\lambda_{1,2}$. The propagation medium is linked to the backscatter medium in (5). The resulting covariances in (6) are used to calculate the following radar measurands of interest:

$$
\begin{array}{rlrl}
Z_{\mathrm{H}} & =10 \log \left(\left\langle\left|S_{a a}\right|^{2}\right\rangle\right) & & \text { H reflectivity, } \\
Z_{\mathrm{dr}} & =10 \log \left[\frac{\left\langle\left|S_{a a}\right|^{2}\right\rangle}{\left\langle\left|S_{b b}\right|^{2}\right\rangle}\right] & \text { Differential reflectivity, } \\
\Psi_{\mathrm{dp}} & =\phi_{\mathrm{dp}}+\delta=\arg \left\{\left\langle S_{b b} S_{a a}^{*}\right\rangle\right\} & \text { Copolar differential phase, } \\
\mathrm{LDR}_{\mathrm{H}}=10 \log \left[\frac{\left\langle\left|S_{a b}\right|^{2}\right\rangle}{\left\langle\left|S_{a a}\right|^{2}\right\rangle}\right] & \text { Linear depolarization ratio (H transmit), }
\end{array}
$$




$$
\begin{array}{rlr}
\mathrm{LDR}_{\mathrm{V}} & =10 \log \left[\frac{\left\langle\left|S_{a b}\right|^{2}\right\rangle}{\left\langle\left|S_{b b}\right|^{2}\right\rangle}\right] & \text { Linear depolarization ratio (V transmit), } \\
\rho_{\mathrm{HV}} & =\frac{\left|\left\langle S_{b b} S_{a a}^{*}\right\rangle\right|}{\left(\left\langle\left|S_{b b}\right|^{2}\right\rangle\left\langle\left|S_{a a}\right|^{2}\right\rangle\right)^{0.5}} & \text { Copolar correlation coefficient, } \\
\rho_{\mathrm{xH}} & =\frac{\left|\left\langle S_{a a} S_{a b}^{*}\right\rangle\right|}{\left(\left\langle\left|S_{a b}\right|^{2}\right\rangle\left\langle\left|S_{a a}\right|^{2}\right\rangle\right)^{0.5}} & \text { Co-to-cross correlation coefficient (H transmit), } \\
\rho_{\mathrm{xV}}=\frac{\left|\left\langle S_{b b} S_{a b}^{*}\right\rangle\right|}{\left(\left\langle\left|S_{a b}\right|^{2}\right\rangle\left\langle\left|S_{b b}\right|^{2}\right\rangle\right)^{0.5}} & \text { Co-to-cross correlation coefficient (V transmit), } \\
\Psi_{\mathrm{xH}}=\phi_{\mathrm{xH}}+\delta_{\mathrm{xH}}=\arg \left\{\left\langle S_{a a} S_{a b}^{*}\right\rangle\right\} & \text { Co-to-cross phase (H transmit), } \\
\Psi_{\mathrm{xV}}=\phi_{\mathrm{xV}}+\delta_{\mathrm{xV}}=\arg \left\{\left\langle S_{b b} S_{a b}^{*}\right\rangle\right\} & \text { Co-to-cross phase (V transmit), }
\end{array}
$$

where $\phi_{\mathrm{dp}}, \phi_{\mathrm{xH}}$, and $\phi_{\mathrm{xV}}$ are due to propagation and $\delta$, $\delta_{\mathrm{xH}}$, and $\delta_{\mathrm{xH}}$ are due to the backscatter medium. ${ }^{1}$ These phases have typically been defined when the propagation medium has a $0^{\circ}$ mean canting angle (i.e., diagonal propagation matrix), and under this assumption the incident polarization on the backscatter medium is either $\mathrm{H}$ or $\mathrm{V}$, assuming $\mathrm{H}$ of $\mathrm{V}$ transmit polarization. Thus, the backscatter and propagation phases are easily separable in the arguments of the cross covariances. When the propagation mean canting angle is nonzero, there is a much more complex relationship between the propagation and backscatter medium. The cross covariances, $\left\langle S_{b b} S_{a a}^{*}\right\rangle,\left\langle S_{a a} S_{a b}^{*}\right\rangle$, and $\left\langle S_{b b} S_{a b}^{*}\right\rangle$, become complicated functions the covariances of the backscatter medium and propagation matrix terms. Nevertheless, we retain the established nomenclature. There is no confusion, for example, by defining $\Psi_{\mathrm{xH}}=\arg \left\{\left\langle S_{a a} S_{a b}^{*}\right\rangle\right\}$. The $\delta_{\mathrm{xH}}$ always is defined as the phase shift upon backscatter for incident pure $\mathrm{H}$ polarization and backscattered $\mathrm{V}$ po- larization. The associated propagation phase is then unambiguously defined as $\phi_{\mathrm{xH}}=\Psi_{\mathrm{xH}}-\delta_{\mathrm{xH}}$. With these definitions in mind, we proceed, but the reader should remember that the cross covariances are complex functions of the backscatter covariances and the propagation parameters when the propagation matrix is nondiagonal. The above-defined radar measurands are plotted as functions of $\phi_{\mathrm{dp}}^{P}$. Unless specified, the frequency is at S band (near $3 \mathrm{GHz}$ ).

\section{Model results}

\section{a. Diagonal propagation matrix}

If the propagation matrix $\mathbf{P}$ is diagonal, the situation is greatly simplified. A diagonal propagation matrix will result when the mean canting angle of the precipitation particles is zero and the distribution of canting angles about the mean is symmetric (Bringi and Chandrasekar 2001). The overall covariance matrix becomes

$$
\boldsymbol{\Sigma}_{0}=\left[\begin{array}{ccc}
|A|^{4}\left\langle\left|S_{\mathrm{HH}}\right|^{2}\right\rangle & \sqrt{2}|A|^{2} A B^{*}\left\langle S_{\mathrm{HH}} S_{\mathrm{HV}}^{*}\right\rangle & A_{2} B^{* 2}\left\langle S_{\mathrm{HH}} S_{\mathrm{Vv}}^{*}\right\rangle \\
\sqrt{2}|A|^{2} B A^{*}\left\langle S_{\mathrm{HV}} S_{\mathrm{HH}}^{*}\right\rangle & 2|A|^{2}|B|^{2}\left\langle\left|S_{\mathrm{HV}}\right|^{2}\right\rangle & \sqrt{2}|B|^{2} A B^{*}\left\langle S_{\mathrm{HV}} S_{\mathrm{Vv}}^{*}\right\rangle \\
B^{2} A^{* 2}\left\langle S_{\mathrm{VV}} S_{\mathrm{HH}}^{*}\right\rangle & \sqrt{2}|B|^{2} B A^{*}\left\langle S_{\mathrm{VV}} S_{\mathrm{HV}}^{*}\right\rangle & |B|^{4}\left\langle\left|S_{\mathrm{VV}}\right|^{2}\right\rangle
\end{array}\right],
$$

where $A$ and $B$ are the propagation parameters $e^{\lambda_{1} z}$ and $e^{\lambda_{2} z}$, respectively. For this case, the backscatter components and the propagation components of the covariances are easily separable. The copolar differential phase [the phase of the $(3,1)$ term in (10)] is routinely estimated (Mueller 1984; Sachidananda and Zrnić 1986) and the separation of propagation phase from backscatter phase can be accomplished using a filtering technique (Hubbert and Bringi 1995). The specific attenu-

\footnotetext{
${ }^{1}$ Note the difference of complex conjugation in the definition here of $\rho_{\mathrm{xH}}$ and $\rho_{\mathrm{xV}}$ as compared to Ryzhkov [2001, his Eqs. (3) and (4)].
}

ation constants may be estimated in several ways that will allow for the correction of the magnitudes of the covariances though this continues to be an area of research (see Bringi and Chandrasekar 2001). The precise values for the specific attenuation constants are not relevant for the following discussion.

The behavior and the possible interpretation of the phases of the co-to-cross covariances $\left(\Psi_{\mathrm{xH}}\right.$ and $\left.\Psi_{\mathrm{xV}}\right)$ are not well documented. If the backscatter medium also has a zero mean canting angle $\alpha$, then the co-to-cross covariances will be zero and the phase of the co-tocross covariances are arbitrary. For nonzero $\alpha$ with 
TABLE 1 . The phases $\delta, \delta_{\mathrm{xH}}$, and $\delta_{\mathrm{xv}}$ as a function of $D_{\mathrm{eq}}$ valid for $0^{\circ}<\alpha<20^{\circ}$ with $\phi_{\mathrm{dp}}^{P}=0^{\circ}$ at $\mathrm{C}$ band (no propagation effects).

\begin{tabular}{ccrc}
\hline \hline$D_{\mathrm{eq}}(\mathrm{mm})$ & $\delta\left(^{\circ}\right)$ & $\delta_{\mathrm{xH}}\left({ }^{\circ}\right)$ & $\delta_{\mathrm{xv}}\left({ }^{\circ}\right)$ \\
\hline 5 & -3.3 & -5.3 & -8.6 \\
6 & 12.6 & 10.4 & 22.9 \\
7 & 23.4 & 22.8 & 46.1 \\
8 & 21.0 & 21.9 & 42.9 \\
\hline
\end{tabular}

$\delta=0$ (e.g., Rayleigh scattering; rain at $\mathrm{S}$ band), the $\Psi_{\mathrm{xH}}$ and $\Psi_{\mathrm{xV}}$ start at zero (positive $\alpha$ ) or $180^{\circ}$ (negative $\alpha$ ) and increase $\left(\Psi_{\mathrm{xV}}\right)$ or decrease $\left(\Psi_{\mathrm{xH}}\right)$ by $0.5 \phi_{\mathrm{dp}}$ as a function of $\phi_{\mathrm{dp}}^{P}$. This is true because there is no depolarization due to the propagation media. But because the $\alpha$ will likely change sign with range (i.e., from resolution volume to resolution volume), both $\Psi_{\mathrm{xH}}$ and $\Psi_{\mathrm{xV}}$ will contain $180^{\circ}$ discontinuities corresponding to the sign changes in $\alpha$ and, thus, plots of $\Psi_{\mathrm{xH}}$ and $\Psi_{\mathrm{xV}}$ versus range will appear noisy. Furthermore, because the magnitudes of $\rho_{\mathrm{xH}}$ and $\rho_{\mathrm{xV}}$ are typically low, the standard measurement error of $\Psi_{\mathrm{xH}}$ and $\Psi_{\mathrm{xV}}$ will be high (see Fig. 1). To eliminate the phase discontinuities caused by the sign change in $\alpha$, the phases $\Psi_{\mathrm{xH}}$ and $\Psi_{\mathrm{xV}}$ are differenced. The model shows that for practically all values of $\alpha$ and $\theta$,

$$
\phi_{X}^{S}=\Psi_{\mathrm{xV}}-\Psi_{\mathrm{xH}}=\Psi_{\mathrm{dp}} .
$$

The $\phi_{X}^{S}$ then serves as a good check as to the quality of the $\Psi_{\mathrm{xH}}$ and $\Psi_{\mathrm{xV}}$ measurements. If $\phi_{X}^{S}$ does not follow $\Psi_{\mathrm{dp}}$, then there are likely problems with the radar system. Again, for this class of scatterers (i.e., Rayleigh, $\left.\delta=0^{\circ}\right), \delta_{\mathrm{xH}}$ and $\delta_{\mathrm{xV}}$ are either 0 or $\pi$, depending on the sign of $\alpha$.

When $\delta$ is nonzero, the $\delta_{\mathrm{xH}}$ and $\delta_{\mathrm{xV}}$ are difficult to estimate accurately from range profiles of $\Psi_{\mathrm{xH}}$ or $\Psi_{\mathrm{xV}}$. The $\Psi_{\mathrm{xH}}\left(\Psi_{\mathrm{xV}}\right)$ will contain a propagation term of $0.5 \phi_{\mathrm{dp}}$ and an additional backscatter phase term. However, it can be shown beginning with (7) that for $\phi_{\mathrm{dp}}^{P}=0$ (i.e., no propagation effects) and with $\alpha<20^{\circ}$ (i.e., $\left\langle S_{\mathrm{HH}} S_{\mathrm{VH}}^{*}\right\rangle,\left\langle S_{\mathrm{VV}} S_{\mathrm{HV}}^{*}\right\rangle$, and $\left\langle\left|S_{\mathrm{HV}}\right|^{2}\right\rangle$ are small compared to other covariances) that

$$
\begin{aligned}
& \delta_{\mathrm{xV}} \approx \tan ^{-1}\left[\frac{\mathfrak{s}\left(\left\langle S_{b b} S_{a a}^{*}\right\rangle\right)}{\mathfrak{R}\left(\left\langle S_{b b} S_{a a}^{*}\right\rangle\right)-\left\langle\left|S_{b b}\right|^{2}\right\rangle}\right] \\
& \delta_{\mathrm{xH}} \approx \tan ^{-1}\left[\frac{\mathfrak{S}\left(\left\langle S_{b b} S_{a a}^{*}\right\rangle\right)}{\left\langle\left|S_{a a}\right|^{2}\right\rangle-\mathfrak{R}\left(\left\langle S_{b b} S_{a a}^{*}\right\rangle\right)}\right],
\end{aligned}
$$

where $\mathfrak{R}$ and $\mathfrak{I}$ denote the real and imaginary parts, respectively. This is a good way to estimate $\delta_{\mathrm{xV}},\left(\delta_{\mathrm{xH}}\right)$ because 1) the copolar signals will have a higher SNR than the cross polar signals and 2) are typically well correlated, and 3 ) the copolar covariances are weak functions of $\theta$. That is, if $\left\langle S_{b b} S_{a a}^{*}\right\rangle,\left\langle\left|S_{b b}\right|^{2}\right\rangle$, and $\left\langle\left|S_{b b}\right|^{2}\right\rangle$ can be accurately estimated for the backscatter medium, then the $\delta_{\mathrm{xH}}$ and $\delta_{\mathrm{xV}}$ can be estimated accurately. This method works well in rain where $\rho_{\mathrm{HV}}$ is high $(>0.95)$ and the

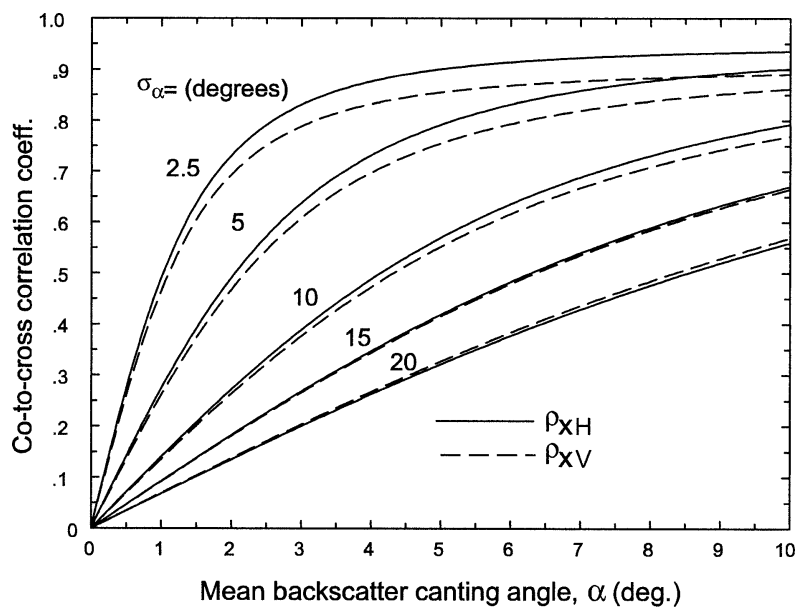

FIG. 2. Co-to-cross correlation coef as a function of mean backscatter canting angle $(\alpha)$ with std $\operatorname{dev}\left(\sigma_{\alpha}\right)$ as a parameter.

LDR is low $(<-25 \mathrm{~dB})$, but may not be accurate in mixed precipitation type regions (i.e., $\rho_{\mathrm{HV}}$ is low) or when $Z_{\mathrm{dr}}$ is close to $0 \mathrm{~dB}$. Also, (12) and (13) are not functions of the sign of the mean canting angle $\alpha$ and thus only the magnitude of the canting angle can be determined from covariance matrices constructed using (12) and (13). The sign of the co-to-cross covariance will change when the sign on the $\alpha$ switches. To illustrate the size of the co-to-cross phases, when $\phi_{\mathrm{dp}}^{P}=0^{\circ}$ at $\mathrm{S}$ band $(\lambda=10$ $\mathrm{cm})$, consider an $8-\mathrm{mm}$ raindrop at $0^{\circ} \mathrm{C}$ with a Pruppacher-Pitter axis ratio. The resulting phases are $\delta=$ $-6.2^{\circ}, \delta_{\mathrm{xH}}=-7.2^{\circ}$, and $\delta_{\mathrm{xV}}=-13.5^{\circ}$. For raindrops with $D_{\text {eq }} \leq 7 \mathrm{~mm}$, where $D_{\text {eq }}$ is the diameter of an equivolumetric sphere, these phase are all nearly zero. At $\mathrm{C}$ band $\left(\lambda=5.5 \mathrm{~cm}, 0^{\circ} \mathrm{C}\right.$, Pruppacher-Pitter axis ratio) these phases are considerably larger. Table 1 gives these phases for $D_{\text {eq }}$ of $5,6,7$, and $8 \mathrm{~mm}$. For such modeled raindrops, the $\delta_{\mathrm{xH}}$ is on the order of $\delta$, while $\delta_{\mathrm{xV}}$ is about twice $\delta$. In general, the relationship among $\delta, \delta_{\mathrm{xH}}$, and $\delta_{\mathrm{xV}}$ is a function of the shape and dielectric constant of the medium (Ryzhkov 2001). However, it can be shown beginning with (12) and (13) that in general $\delta_{\mathrm{xV}}-\delta_{\mathrm{xH}}$ $=\delta$ if $\rho_{\mathrm{HV}}$ is high. This relationship is consistent with (11). Note the sign difference between the angles of Table 1 and those in Ryzhkov's (2001) Fig. 7, which are incorrect (Ryzhkov 2002, personal communication).

Next, consider the effect of nonzero $\alpha$ on the magnitude of the co-to-cross covariances. The backscatter medium is modeled as rain with an exponential size distribution with parameters of $D_{\max }=8 \mathrm{~mm}, D_{0}=$ $2.56 \mathrm{~mm}$, and $N_{0}=8000 \mathrm{~mm}^{-1} \mathrm{~m}^{-1}$. The orientation distribution is Fisher (Mardia 1972; Hubbert and Bringi 1996). Figure 2 shows $\rho_{\mathrm{xH}}$ (solid lines) and $\rho_{\mathrm{xV}}$ (dashed lines) as a function of the mean backscatter canting angle $(\alpha)$ with the standard deviation $\left(\sigma_{\alpha}\right)$ as a parameter. As the $\sigma_{\alpha}$ increases, $\rho_{\mathrm{xH}}$ and $\rho_{\mathrm{xV}}$ become less sensitive to $\alpha$. In light stratiform rain with low turbulence, where one would expect a small $\sigma_{\alpha}, \rho_{\mathrm{xH}}$, and $\rho_{\mathrm{xV}}$ could 


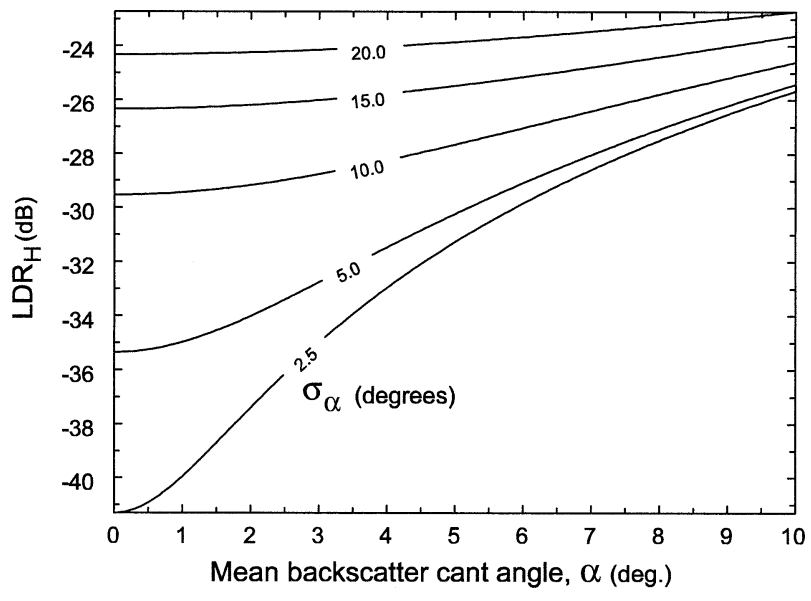

FIG. 3. $\operatorname{LDR}_{\mathrm{H}}$ as a function of mean backscatter canting angle $(\alpha)$ with the std dev of $\left(\sigma_{\alpha}\right)$ as a parameter.

be large with just a small $\alpha\left[1.5^{\circ}\right.$ gives $\rho_{\mathrm{xH}}\left(\rho_{\mathrm{xV}}\right) \approx$ 0.63]. In a convective rain environment with more turbulence, one might expect larger $\sigma_{\alpha}$ of about $10^{\circ}$ (Hubbert and Bringi 2001). In this case $\alpha=1.5^{\circ}$ gives $\rho_{\mathrm{xH}}$ $\left(\rho_{\mathrm{xV}}\right) \approx 0.2$ and $\alpha=5^{\circ}$ gives $\rho_{\mathrm{xH}}\left(\rho_{\mathrm{xV}}\right) \approx 0.42$. Figure 3 shows the effect of $\alpha$ on $\operatorname{LDR}_{\mathrm{H}}$ with $\sigma_{\alpha}$ as a parameter. As expected, $\mathrm{LDR}_{\mathrm{H}}$ increases with increasing $\sigma_{\alpha}$. Presently, the CSU-CHILL radar's LDR system limit is from around -33 to $-34 \mathrm{~dB}$; thus, detecting raindrop distributions with $\sigma_{\alpha}<5^{\circ}$ and $\alpha<3^{\circ}$ is impossible either with LDR or with $\rho_{\mathrm{xH}}\left(\rho_{\mathrm{xV}}\right)$ for the case of the zero propagation phase.

\section{b. Nonzero mean propagation canting angle}

The separation of propagation and backscatter terms in the covariances becomes quite difficult when both $\alpha$ and $\theta$ are nonzero (recall that $\alpha$ refers to the backscatter medium and $\theta$ refers to the propagation medium) and thus the numerical model is necessary for the most accurate modeling. The backscatter medium is modeled as rain as above and with $\sigma_{\alpha}=10^{\circ}$. The propagation matrix is assumed to have absolute and specific attenuation of $A_{\mathrm{H}} / K_{\mathrm{dp}}=0.0165 \mathrm{~dB} \mathrm{deg}-1$ and $A_{\mathrm{dp}} / K_{\mathrm{dp}}=0.004$ $\mathrm{dB} \mathrm{deg}^{-1}$, which are typical values for $\mathrm{S}$ band (Bringi et al. 1990). The model demonstrates that the conventional radar variables, $Z_{\mathrm{H}}, Z_{\mathrm{dr}}, \phi_{\mathrm{dp}}$, and $\rho_{\mathrm{HV}}$ are insensitive to mean propagation canting angles $(\theta)$, less than about $10^{\circ}$ for the parameters selected here. For $\theta=10^{\circ}$ and $\phi_{\mathrm{dp}}^{P}$ of $180^{\circ}$, the $Z_{\mathrm{H}}$ and $Z_{\mathrm{dr}}$ deviate less than 0.5 $\mathrm{dBZ}$ and $0.2 \mathrm{~dB}$, respectively, from their nominal values (i.e., when $\phi_{\mathrm{dp}}^{P}=0^{\circ}$ ). At $\phi_{\mathrm{dp}}^{P}=90^{\circ}$ with $\theta=10^{\circ}, \phi_{\mathrm{dp}}$ is about $86^{\circ}$. The $\rho_{\mathrm{HV}}$ changes less than 0.002 from its nominal value. Nonzero $\theta$ does, however, dramatically change the cross polar power and the co-to-cross covariances. Shown in Figs. 4 and 5 are $\mathrm{LDR}_{\mathrm{H}}$ and $\rho_{\mathrm{xH}}$ as functions of the principal plane copolar differential phase $\phi_{\mathrm{dp}}^{P}$ with the mean canting angle of the propagation medium $\theta$ as a parameter. For simplicity, the

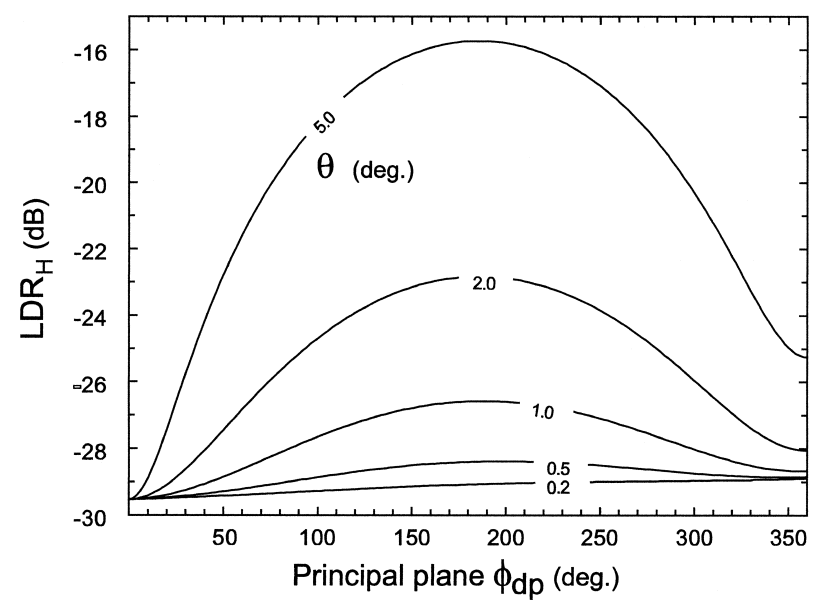

FIG. 4. $\mathrm{LDR}_{\mathrm{H}}$ as functioin of principal plane $\phi_{\mathrm{dp}}$ with mean propagation canting angle $\theta$ as a parameter. The mean backscatter canting angle $\alpha$ is $0^{\circ}$.

mean backscatter canting angle is $0^{\circ}$. For $\theta<0.5^{\circ}$, the effect on $\mathrm{LDR}_{\mathrm{H}}$ is minimal with $\mathrm{LDR}_{\mathrm{H}}$ increasing by less than $1 \mathrm{~dB}$ from its nominal value (nominal value closely follows the curve marked $0.2^{\circ}$ ) after about $150^{\circ}$ of $\phi_{\mathrm{dp}}^{P}$. The increasing trend of $\mathrm{LDR}_{\mathrm{H}}$ seen in the curve labeled $0.2^{\circ}$ is caused by the assumed differential attenuation. The effect of $\theta$ on $\rho_{\mathrm{xH}}$ is more accentuated with $\rho_{\mathrm{xH}}$ increasing to more than 0.3 from the nominal value of zero after $100^{\circ}$ of $\phi_{\mathrm{dp}}^{P}$ for $\theta \geq 0.5^{\circ}$. If $\sigma_{\alpha}$ is less than $10^{\circ}$, the effect of $\theta$ on $\operatorname{LDR}_{\mathrm{H}}$ and $\rho_{\mathrm{xH}}$ is greater. For example, the case where $\sigma_{\alpha}=5^{\circ}$ was shown in Hubbert et al. (1999, see their Fig. 1, 2). This then suggests a way to check for the presence of nonzero $\theta$ in experimental data. If obvious increasing trends in $\rho_{\mathrm{xH}}$ and $\mathrm{LDR}_{\mathrm{H}}$ range profiles are detected along paths where there is large accumulation of $\phi_{\mathrm{dp}}$, then nonzero $\theta$ is likely present. Polarization errors can also cause such effects and are discussed later.

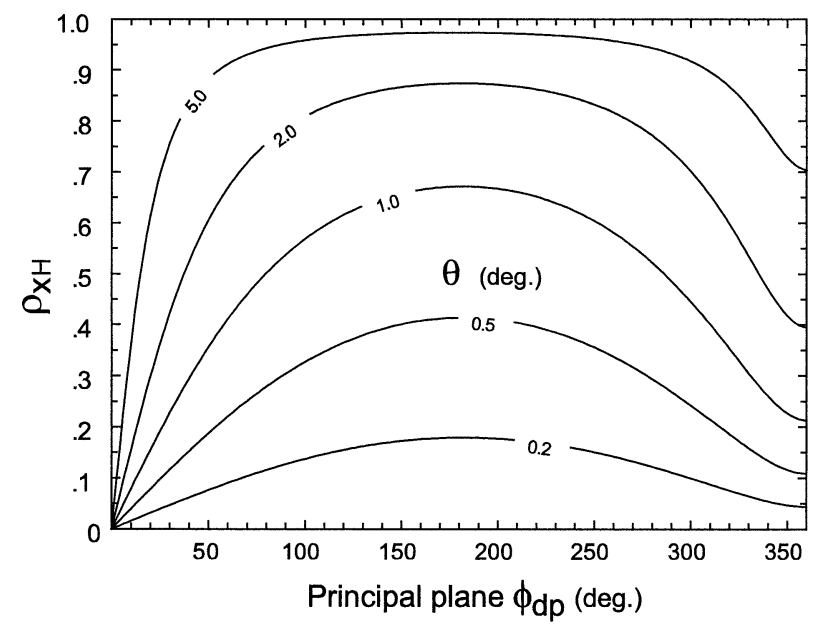

FIG. 5. Same as in Fig. 4, but for the co-to-cross correlation coefficient $\rho_{\mathrm{xH}}$. 


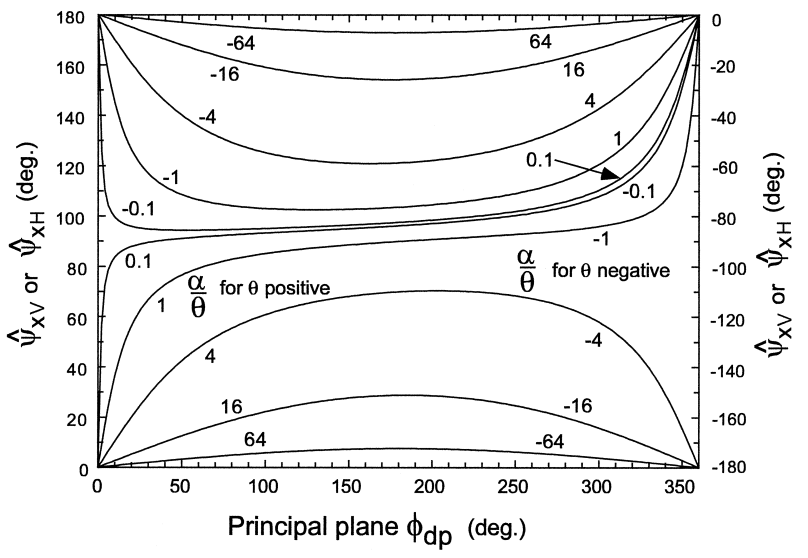

FIG. 6. The phases $\hat{\Psi}_{\mathrm{xV}}$ and $\hat{\Psi}_{\mathrm{xH}}$ as a function of $\phi_{\mathrm{dp}}^{P}$ with the ratio $\alpha / \theta$ as a parameter. The left-hand axis corresponds to positive values of $\theta$, while the right-hand vertical axis corresponds to negative values of $\theta$

The effect of nonzero $\theta$ on the co-to-cross phases $\Psi_{\mathrm{xH}}$ and $\Psi_{\mathrm{xV}}$ is particularly complicated, especially in conjunction with nonzero $\alpha$. However, the analysis is simplified by making a few observations. First, because $\Psi_{\mathrm{xV}}$ $-\Psi_{\mathrm{xH}}=\Psi_{\mathrm{dp}}$, this suggests creating the following two new phase variables:

$$
\begin{aligned}
& \hat{\Psi}_{\mathrm{xH}}=\Psi_{\mathrm{xH}}+\Psi_{\mathrm{dp}} / 2, \\
& \hat{\Psi}_{\mathrm{xV}}=\Psi_{\mathrm{xV}}-\Psi_{\mathrm{dp}} / 2 .
\end{aligned}
$$

It appears that $\Psi_{\mathrm{xH}}$ and $\Psi_{\mathrm{xH}}$ are corrected for propagation phase; however, the resulting phases, $\hat{\Psi}_{\mathrm{xH}}$ and $\hat{\Psi}_{\mathrm{xH}}$, are not to be interpreted as backscatter phases, that is, $\hat{\Psi}_{\mathrm{xH}}$ $\neq \delta_{\mathrm{xH}}$ and $\hat{\Psi}_{\mathrm{xV}} \neq \delta_{\mathrm{xV}}$. Interestingly, the model shows that $\hat{\Psi}_{\mathrm{xH}}$ and $\hat{\Psi}_{\mathrm{xV}}$ are nearly identical functions of $\phi_{\mathrm{dp}}^{P}$ with the restrictions $\alpha<25^{\circ}$ and $\theta<10^{\circ}$. In addition, the model shows that the behavior of $\hat{\Psi}_{\mathrm{xH}}$ and $\hat{\Psi}_{\mathrm{xV}}$ is determined by the ratio of the backscatter and propagation mean canting angles $\alpha / \theta$. Figure 6 shows $\hat{\Psi}_{\mathrm{xV}}$ and $\hat{\Psi}_{\mathrm{xV}}$ as functions of $\phi_{\mathrm{dp}}^{P}$ with the ratio of $\alpha / \theta$ as a parameter. The left-hand axis in Fig. 6 is for positive $\theta$ and for $\alpha / \theta$ values shown on the left-hand part of the graph. All curves lie between $0^{\circ}$ and $180^{\circ}$. The righthand axis is for negative $\theta$ and for $\alpha / \theta$ values shown on the right-hand part of the graph. All curves lie between $0^{\circ}$ and $-180^{\circ}$. Thus, if experimental curves of $\hat{\Psi}_{\mathrm{xH}}\left(\hat{\Psi}_{\mathrm{xV}}\right)$ are strictly positive (negative) this would either indicate a mean positive (negative) $\theta$ is present or again could indicate the presence of polarization errors. As Fig. 6 shows, if the $\theta$ and $\alpha$ are small (as expected in rain) with a ratio of about 1 , then the $\hat{\Psi}_{\mathrm{xH}}$ and $\hat{\Psi}_{\mathrm{xV}}$ will cluster around $\pm 90^{\circ}$ after a few tens of degrees of $\phi_{\mathrm{dp}}$ are accumulated. In the experimental section this is shown to be the case for the CSU-CHILL radar data collected during the Severe Thunderstorm Electrification and Precipitation Study (STEPS).

\section{Polarization errors}

\section{Theory}

The ideal two-channel H/V system would radiate alternately pure $\mathrm{H}$ and $\mathrm{V}$ polarized electromagnetic waves and then also receive pure $\mathrm{H}$ and $\mathrm{V}$ polarized components of the scattered wave. In practice, polarization errors are introduced by cross coupling in the radar microwave circuitry and in imperfections in the polarization purity of the antenna pattern, which can vary throughout the beam, especially where cross-polarized lobes exist (Bringi and Chandrasekar 2001). For distributed precipitation media, the resulting error is an integrated effect. Polarization errors have been covered in detail by McCormick (1981) whose treatment was analytical and was applied primarily to circular polarization basis. Here, the polarization errors are integrated and represented by a single complex error term for each channel. The polarization errors are easily included in the model by pre- and postmultiplying $\mathbf{S}$ of (5) by the error matrix $\mathbf{Y}$,

$$
\mathbf{S}_{e}=\mathbf{Y}^{\mathrm{T}} \mathbf{S} \mathbf{Y}
$$

where

$$
\mathbf{Y}=\left[\begin{array}{ll}
i_{\mathrm{H}} & \varepsilon_{\mathrm{V}} \\
\varepsilon_{\mathrm{H}} & i_{\mathrm{V}}
\end{array}\right],
$$

with constraints $i_{\mathrm{H}}^{2}+\left|\varepsilon_{\mathrm{H}}\right|^{2}=i_{\mathrm{V}}^{2}+\left|\varepsilon_{\mathrm{V}}\right|^{2}=1$ where $i_{\mathrm{H}}$ and $i_{\mathrm{V}}$ are real. The polarization errors of the $\mathrm{H}$ and $\mathrm{V}$ channels are represented by the complex numbers $\varepsilon_{\mathrm{H}}$ and $\varepsilon_{\mathrm{V}}$, respectively. The polarization errors can also be equivalently represented with the geometric ellipse parameters of tilt angle $\tau$ and ellipticity angle $\epsilon$. These variables are related by (Azzam and Bashara 1989)

$$
\begin{aligned}
& \tan 2 \tau=\frac{2 \mathfrak{R}(\chi)}{1-|\chi|^{2}} \\
& \sin 2 \epsilon=\frac{2 \Im(\chi)}{1+|\chi|^{2}},
\end{aligned}
$$

where $\chi$ is the polarization ratio given in (9) and $\mathfrak{R}$ and $\mathfrak{s}$ denote real and imaginary parts, respectively. For $\mathrm{H}$ errors, $\chi=\varepsilon_{\mathrm{H}} / i_{\mathrm{H}}$ and for $\mathrm{V}$ errors, $\chi=i_{\mathrm{V}} / \varepsilon_{\mathrm{V}}$. As can be seen from the equations, if the $\varepsilon_{\mathrm{H}}\left(\varepsilon_{\mathrm{V}}\right)$ is real then $\epsilon$ is zero, and if $\varepsilon_{\mathrm{H}}\left(\varepsilon_{\mathrm{V}}\right)$ is imaginary then $\tau$ is zero. If the errors are orthogonal, that is, $\varepsilon_{\mathrm{V}}=-\varepsilon_{\mathrm{H}}^{*}$, then $\mathbf{Y}$ is unitary and (16) represents an orthogonal change of polarization basis. Separating the polarization errors into their geometric components gives a convenient and intuitive way to analyze polarization errors. Because the copolar covariances are negligibly affected by small polarization errors, we next investigate the effect of polarization errors on just the covariances involving the cross polar signals. Figures 7 and 8 show the effect of tilt angle error on $\mathrm{LDR}_{\mathrm{H}}$ and $\rho_{\mathrm{xH}}$, with $\sigma_{\alpha}$ as a parameter for $\phi_{\mathrm{dp}}^{P}=0$. For $|\tau|<0.5^{\circ}, \rho_{\mathrm{xH}}$ reaches a maximum of 0.15 when $\sigma_{\alpha}=5^{\circ}$ and is always below 0.1 when 


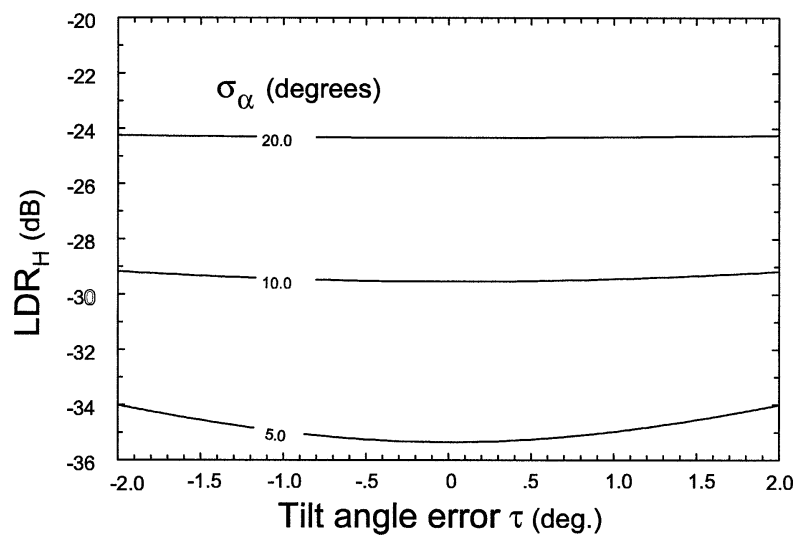

FIG. 7. $\mathrm{LDR}_{\mathrm{H}}$ as a function of tilt angle error with $\sigma_{\alpha}$ as a parameter and $\phi_{\mathrm{dp}}^{P}=0^{\circ}$.

$\sigma_{\alpha}>10^{\circ}$. The $\mathrm{LDR}_{\mathrm{H}}$ is much less affected by tilt angle errors remaining nearly constant for $\sigma_{\alpha}=10^{\circ}, 20^{\circ}$ and varying only a little more than $1 \mathrm{~dB}$ for $\sigma_{\alpha}=5^{\circ}$. Obviously, $\mathrm{LDR}_{\mathrm{H}}$ is much more sensitive to $\sigma_{\alpha}$ than to $\tau$ for the range of values selected. The curves in Figs. 7 and 8 are identical to portions of the curves shown in Figs. 2 and 3. This occurs because tilt errors, when defined as above, produce the same effect as $\alpha$; that is, $\tau=2^{\circ}$ is identical to $\alpha=-2^{\circ}$ in terms of the resulting radar signatures. Figures 9 and 10 show the effect of ellipticity angle error $\epsilon$ on $\mathrm{LDR}_{\mathrm{H}}$ and $\rho_{\mathrm{xH}}$ with $\sigma_{\alpha}$ as a parameter. Both $\mathrm{LDR}_{\mathrm{H}}$ and $\rho_{\mathrm{xH}}$ are much more sensitive to ellipticity errors than tilt errors and the sensitivity increases for decreasing $\sigma_{\alpha}$. As can be seen, for a constant error, as $\sigma_{\alpha}$ decreases $\mathrm{LDR}_{\mathrm{H}}$ decreases, but $\rho_{\mathrm{xH}}$ increases. For example, in light stratiform rain where raindrops are likely well aligned with a small $\sigma_{\alpha}$, if there is a small tilt angle or ellipticity angle error, LDR can be very low while $\rho_{\mathrm{xV}}$ and $\rho_{\mathrm{xH}}$ can be quite high.

The co-to-cross phases $\Psi_{\mathrm{xH}}$ and $\Psi_{\mathrm{xV}}$ are very sensitive to polarization errors and understanding these effects is essential in determining the calibration offset phases

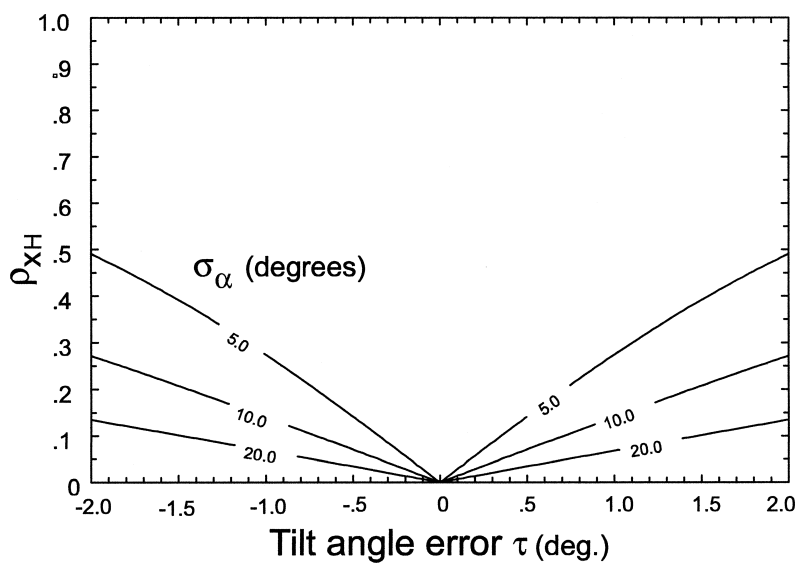

FIG. 8. The $\rho_{\mathrm{xH}}$ as a function of tilt angle error with $\sigma_{\alpha}$ as a parameter and $\phi_{\mathrm{dp}}^{p}=0^{\circ}$.

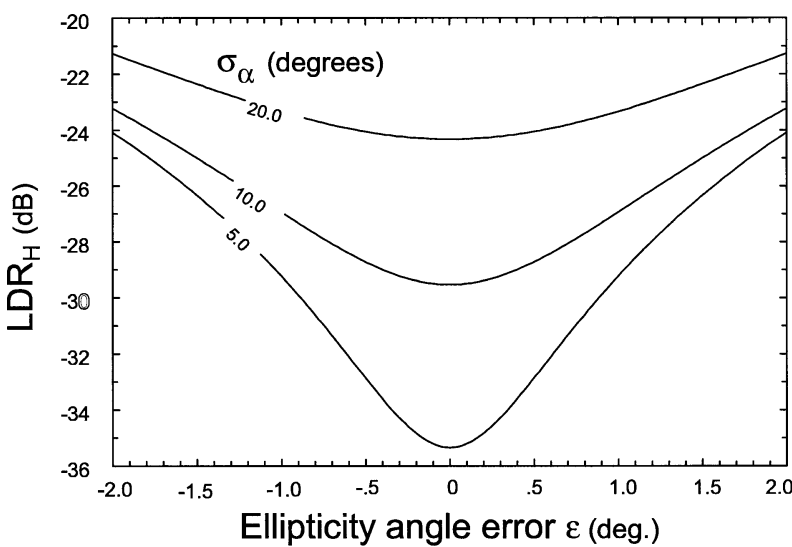

FIG. 9. The $\mathrm{LDR}_{\mathrm{H}}$ as a function of ellipticity angle error with $\sigma_{\alpha}$ as a parameter and $\phi_{\mathrm{dp}}^{P}=0^{\circ}$.

(see Part I). If there are no polarization errors and the propagation matrix is diagonal, then $\Psi_{\mathrm{xH}}\left(\Psi_{\mathrm{xV}}\right)$ start at $0^{\circ}$ and decrease (increase) as $\phi_{\mathrm{dp}} / 2$ is a function of $\phi_{\mathrm{dp}}^{P}$. If the polarization errors are real only (i.e., tilt angle error with no ellipticity angle error), then the phases $\Psi_{\mathrm{xH}}$ and $\Psi_{\mathrm{xV}}$ are zero when $\phi_{\mathrm{dp}}$ is zero. In other words, tilt angle errors do not affect the offset phase of $\Psi_{\mathrm{xH}}$ and $\Psi_{\mathrm{xV}}$. If the polarization errors are imaginary only (i.e., ellipticity angle error with no tilt angle error), then the phases $\Psi_{\mathrm{xH}}$ and $\Psi_{\mathrm{xV}}$ are offset by $\pm 90^{\circ}$ when $\phi_{\mathrm{dp}}$ is zero. When the polarization errors are complex (i.e., both tilt and ellipticity angle errors are present), the phase offsets of $\Psi_{\mathrm{xH}}$ and $\Psi_{\mathrm{xV}}$ can be any angle but they will be equal so that the condition $\Psi_{\mathrm{xV}}-\Psi_{\mathrm{xH}} \approx \phi_{\mathrm{dp}}$ (11) still generally holds true.There are some discrepancies depending on the polarization errors, but these deviations are very small and (11) remains a very good approximation.

\section{Estimating polarization errors from data}

To construct covariance matrices that are accurate representations of the scattering medium, first the mag-

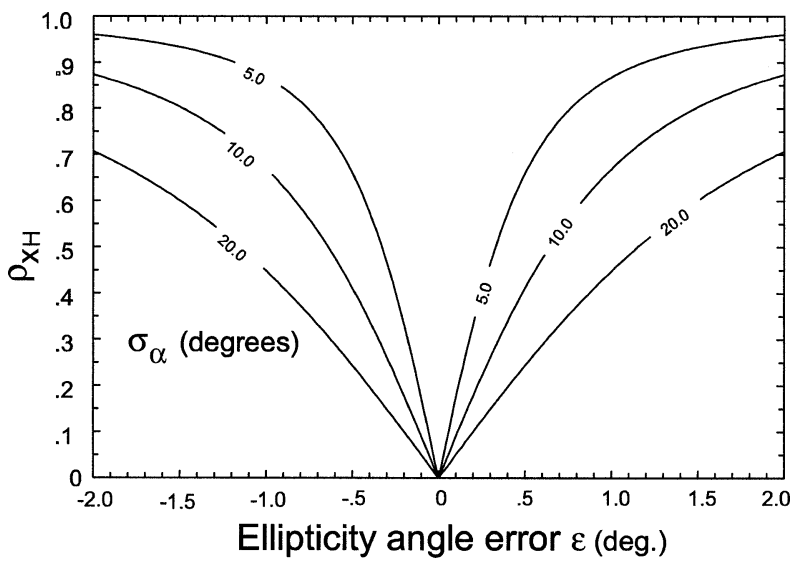

FIG. 10. The $\rho_{\mathrm{xH}}$ as a function of ellipticity angle error with $\sigma_{\alpha}$ as a parameter and $\phi_{\mathrm{dp}}^{P}=0^{\circ}$. 
nitude and phase biases due to the radar system need to be assessed as was done in Part I. That model, however, did not account for the possible presence of polarization errors that can affect the $\Psi_{\mathrm{xH}}$ and $\Psi_{\mathrm{xV}}$ greatly. Inclusion of polarization errors affects the phase offsets of the various interchannel covariances and further adjustments to the phase offset estimates, as given in Part I, may be required.

Evidence of phase offset due to polarization errors is found in the ice phase of storms where the mean canting angle of the precipitation in the backscatter resolution volume should be on average zero. There are circumstances where nonzero mean canting can be found in the ice phase, that is, due to electric fields (Caylor and Chandrasekar 1996), and these areas need to be avoided for the following technique to be valid. It is possible to determine the mean canting angle of the particles in the backscatter resolution volume from optimum polarizations determined via matrix methods (Tragl et al. 1991). Using this method, range profiles of mean canting angle in the ice phase of a convective storm can be constructed where very little or no propagation effects are present (e.g., no differential propagation phase or attenuation is present). If the average mean canting angle of all of the resolution volumes is not approximately zero, then the phases $\Psi_{\mathrm{xH}}$ and $\Psi_{\mathrm{xV}}$ can be adjusted by $\phi_{\mathrm{err}}$ as

$$
\begin{aligned}
& \Psi_{\mathrm{xH}}^{e}=\Psi_{\mathrm{xH}}-\phi_{x_{\mathrm{O}} \mathrm{fff}}+\phi_{\mathrm{err}}, \\
& \Psi_{\mathrm{xV}}^{e}=\Psi_{\mathrm{xV}}+\phi_{x_{-} \text {off }}+\phi_{\mathrm{err}},
\end{aligned}
$$

until the average of the mean canting angles is zero. The $\phi_{x_{\text {-off }}}$ is estimated as discussed in Part I of this paper. This then completes the process of calibrating covariance matrices for the purpose of estimating polarization errors.

The polarization error estimation method is based on the premise that the sum of $\rho_{\mathrm{xV}}$ or $\rho_{\mathrm{xH}}$ along a range profile in rain will be minimum when the polarization basis is $\mathrm{H} / \mathrm{V}$. It is assumed that the mean canting angle of the propagation medium will be, on average, zero. If the polarization basis is $\mathrm{H} / \mathrm{V}$, then no depolarization occurs along the propagation path and $\rho_{\mathrm{xV}}$ and $\rho_{\mathrm{xH}}$ are determined solely by the backscatter medium. The model shows that if there is a mean canting angle of the propagation medium, then there will be depolarization along the propagation path and this will in general cause $\rho_{\mathrm{xV}}$ and $\rho_{\mathrm{xH}}$ to increase and be a function of $\phi_{\mathrm{dp}}$. Polarization errors effectively cause the same phenomena. With polarization errors present, depolarization occurs along the propagation path and in general this causes $\rho_{\mathrm{xV}}$ and $\rho_{\mathrm{xH}}$ to be higher than if the polarization basis were pure $\mathrm{H} / \mathrm{V}$. Thus, in general, polarization errors will increase the average value of the $\rho_{\mathrm{xV}}$ or $\rho_{\mathrm{xH}}$ over a range profile where significant $\left(>90^{\circ}\right) \phi_{\mathrm{dp}}$ accumulation is present. In order to observe any systematic shifts in $\rho_{\mathrm{xV}}$ or $\rho_{\mathrm{xH}}$ along range profiles, it is necessary to have significant accumulation of $\phi_{\mathrm{dp}}$. Depending upon the polarization errors, the range profiles of $\rho_{\mathrm{xV}}$ or $\rho_{\mathrm{xH}}$ can in- crease or decrease as a function of $\phi_{\mathrm{dp}}$ achieving maximums or minimums for various values of $\phi_{\mathrm{dp}}$. But in any event, the average value of $\rho_{\mathrm{xV}}$ or $\rho_{\mathrm{xH}}$ over a significant range of accumulated $\phi_{\mathrm{dp}}$ will be minimized in the $\mathrm{H} / \mathrm{V}$ polarization basis. For each individual resolution volume, the covariance matrix will yield a nonzero tilt and ellipticity angle that minimizes $\rho_{\mathrm{xV}}$ or $\rho_{\mathrm{xH}}$, but the mean value of $\rho_{\mathrm{xV}}$ or $\rho_{\mathrm{xH}}$ over range will be minimized when the polarization basis is $\mathrm{H} / \mathrm{V}$. This is the basis of the polarization error determination method. Thus, if $\rho_{\mathrm{xV}}$ and $\rho_{\mathrm{xH}}$ are high in rain data, then it is reasonable to assume that polarization errors are the cause barring other known anomalies (e.g., interference, three-body scattering, second trip echos, radar malfunction). Finding error terms that minimize $\rho_{\mathrm{xV}}$ or $\rho_{\mathrm{xH}}$ should yield unique polarization errors because eigenpolarizations (or optimum polarizations) of the propagation medium are unique (i.e., the wave polarizations that pass through the medium without depolarization; Azzam and Bashara 1989). Instead of minimizing the cross covariance, there is a more general condition for optimum polarizations. From the general covariance matrix theory (Tragl 1990; Tragl et al. 1991; Lüneburg et al. 1991), optimum polarizations for distributed scatterers are solutions to

$$
\left\langle S_{a a} S_{a b}^{*}(\chi)\right\rangle-\left\langle S_{b b}^{*} S_{a b}(\chi)\right\rangle=0,
$$

where $\chi$ is polarization ratio variable as given in (7). The tilt angle of the characteristic optimum polarization basis (corresponding do the smallest eigenvalue) gives the mean canting angle of the precipitation medium (if no polarization errors are present) and can be found via analytical matrix methods (Tragl et al. 1991; Lüneburg et al. 1991), which are based on orthogonal basis transformations. However, polarization errors can be nonorthogonal, so that finding the tilt and ellipticity angles via the above-mentioned analytical method may not reveal the true error terms. Thus, the errors here are determined by finding values of the error terms, $\tau_{\mathrm{H}}, \epsilon_{\mathrm{H}}$, $\tau_{\mathrm{V}}, \epsilon_{\mathrm{V}}$, that minimize $\left\langle S_{a a} S_{a b}^{*}\right\rangle-\left\langle S_{b b}^{*} S_{a b}\right\rangle$, and this is done via a simple search method.

The mechanics of the polarization error estimation method are as follows. A range profile(s) of data is selected where there is significant increase in $\Psi_{\mathrm{dp}}$, which will accentuate the errors thus making it possible to detect small polarization errors. For example, if significant tilt error is present (a few tenths of a degree is sufficient), then in general $\rho_{\mathrm{xH}}$ and $\rho_{\mathrm{xV}}$ will increase significantly with increasing $\Psi_{\mathrm{dp}}$. Calibrated covariance matrices are constructed at each sample point (spaced at $0.15 \mathrm{~km}$ in the following case). For all the selected covariance matrices, error terms $\tau_{\mathrm{H}}, \tau_{\mathrm{V}}, \epsilon_{\mathrm{H}}$, and $\epsilon_{\mathrm{V}}$, are varied and the minimum of the sum

$$
\begin{aligned}
\Omega= & \sum_{i=1}^{N}\left\langle S_{a a} S_{a b}^{*}\left(i ; \tau_{\mathrm{H}}, \tau_{\mathrm{V}}, \epsilon_{\mathrm{H}}, \epsilon_{\mathrm{V}}\right)\right\rangle \\
& -\left\langle S_{b b}^{*} S_{a b}\left(i ; \tau_{\mathrm{H}}, \tau_{\mathrm{V}}, \epsilon_{\mathrm{H}}, \epsilon_{\mathrm{V}}\right)\right\rangle
\end{aligned}
$$




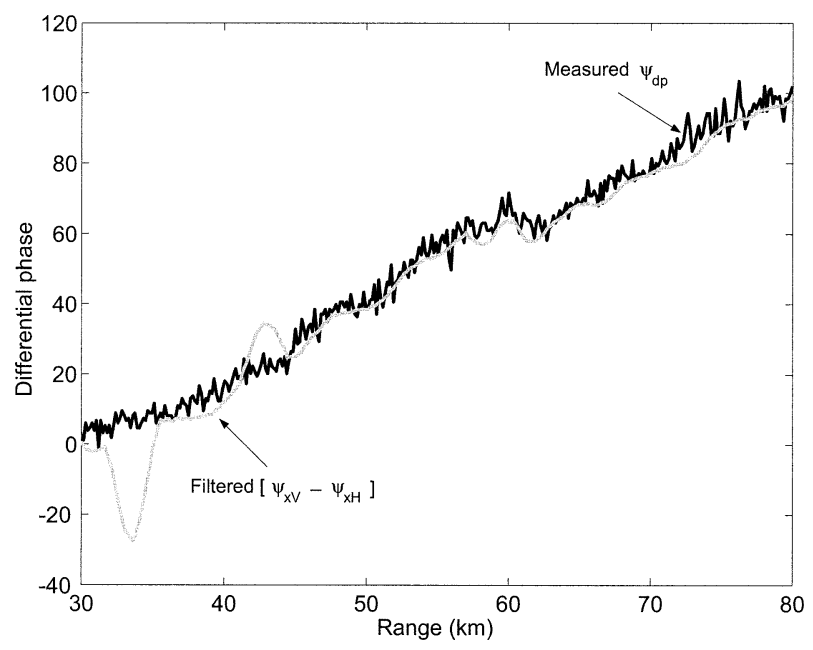

FIG. 11. Range profile of $\Psi_{\mathrm{dp}}$ and $\Psi_{\mathrm{xV}}-\Psi_{\mathrm{xH}}$.

is found where $N$ is the total number of covariance matrices. The resulting tilt and ellipticity angles are considered the integrated polarization errors.

\section{Data analysis}

Shown in Figs. 11-14 is a ray of data gathered with the CSU-CHILL radar (Brunkow et al. 2000) during STEPS on 21 July 2000 through a heavy rain cell with reflectivities of 40-60 dBZ. Figure 11 shows $\Psi_{\mathrm{dp}}$ increasing $100^{\circ}$ over $50 \mathrm{~km}$ and a filtered version of $\Psi_{\mathrm{xV}}$ $-\Psi_{\mathrm{xH}}$ which mimics $\Psi_{\mathrm{dp}}$ very well, as expected. Figure 12 shows a range profile of $\hat{\Psi}_{\mathrm{xV}}$ [see (15)] with 150-m range sampling. According to the model, if the mean propagation canting angle is zero, then this phase should be $0^{\circ}$ or $180^{\circ}$ (assuming $\delta=0^{\circ}$ ), depending on the mean canting angle of the backscatter resolution volume. Because this phase is nearly always positive, we surmise from Fig. 6 that $\theta$ must be positive or, more likely, that polarization errors are present. Thus, if the mean canting angle of the propagation medium is zero, then there is likely negative polarization tilt error present (tilt error $\tau$ is opposite in sign to $\theta$ ). Figure 12 is not a single ray anomaly but rather is seen in nearly all range profiles of CSU-CHILL data gathered during STEPS that possess large amounts of increasing $\Psi_{\mathrm{dp}}$. There are 333 covariance matrices available through the region of large phase accumulation that are used in the minimization procedure (23). The resulting polarization errors are $\tau_{\mathrm{H}}=-0.5^{\circ}, \epsilon_{\mathrm{H}}=-0.1^{\circ}, \tau_{\mathrm{V}}=89.5^{\circ}$, and $\epsilon_{\mathrm{V}}=0.4^{\circ}$. Using these error terms, a $3 \times 3$ transformation matrix can be constructed and the polarization errors can be removed from the data by pre- and post multiplying the measured covariance matrices by this matrix (Huang et al. 2001). Range profiles of the corrected data can then be made. Figure 13 shows measured $\rho_{\mathrm{xH}}$ and corrected $\rho_{\mathrm{xH}}$ and, as expected, corrected $\rho_{\mathrm{xH}}$ is significantly reduced to an average level that is consistent with rain.

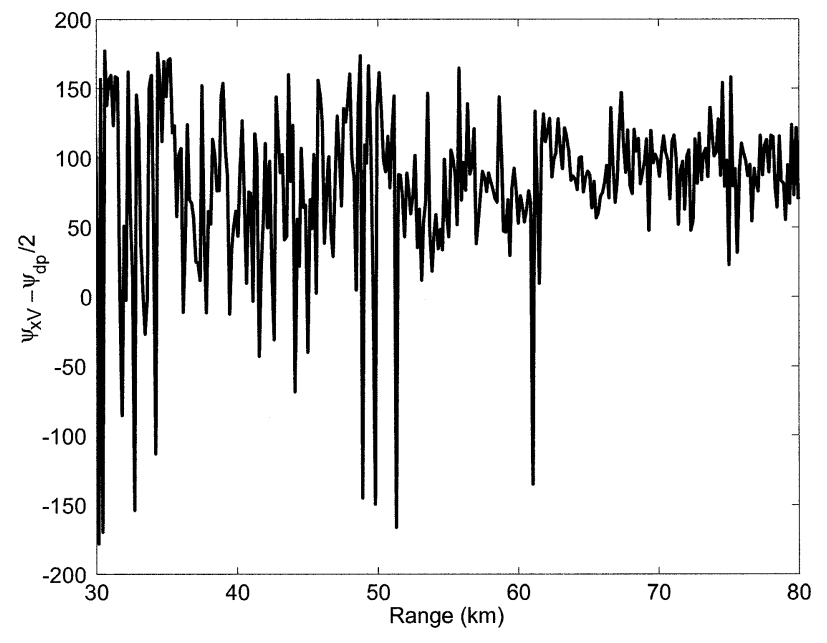

FIG. 12. Range profile of $\Psi_{\mathrm{xV}}-\Psi_{\mathrm{dp}} / 2$.

Similarly, Fig. 14 shows filtered and corrected LDR. Again, the the corrected LDR is reduced as it should be with the polarization errors removed. As an internal consistency check as to the validity of the estimated polarization errors, the error terms are next put into the scattering model. The canting angles $\theta$ and $\alpha$ are set to zero and the canting angle distribution is Fisher where $\sigma_{\alpha}$ is $10^{\circ}$. Figure 15a shows the resulting $\rho_{\mathrm{xH}}$ (solid line) and $\mathrm{LDR}_{\mathrm{H}}$ (dashed line) while Fig. $15 \mathrm{~b}$ shows $\hat{\Psi}_{\mathrm{xH}}$, all as functions of principal plane $\phi_{\mathrm{dp}}$. When comparing these figures to the experimental data shown in Figs. $12-14$, it can be seen that the trends and general behavior predicted by the model are also observed in the data.

\section{Summary and conclusions}

A complete numerical covariance matrix model is described where the parameters of the propagation and

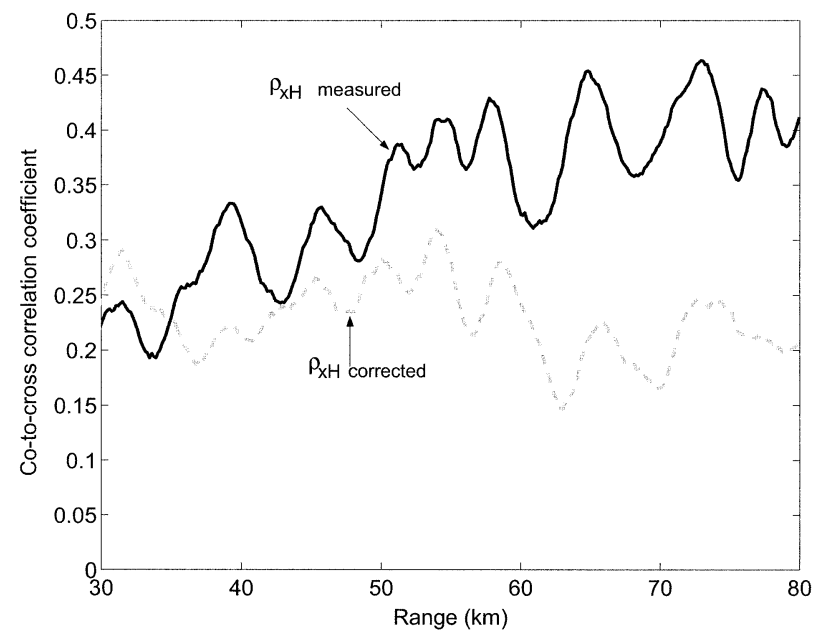

FIG. 13. Range profile of filtered $\rho_{\mathrm{xH}}$ and polarization error corrected $\rho_{\mathrm{xH}}$. 


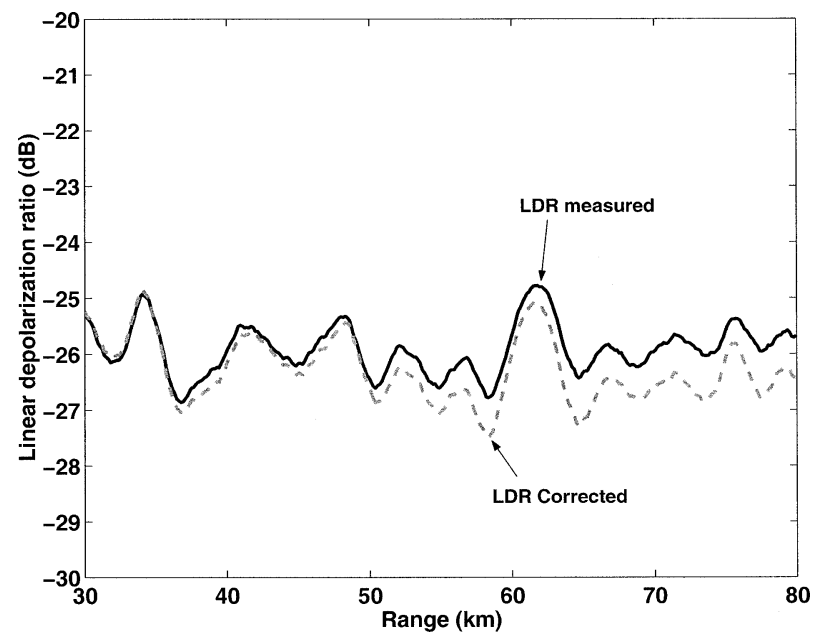

FIG. 14. Range profile of filtered LDR and polarization error corrected LDR.

backscatter medium can be independently varied. Polarization errors are also included. The propagation medium was modeled via a $2 \times 2$ matrix (i.e., coherent scattering) and the backscatter medium was modeled by a covariance matrix constructed by the T-matrix method for an ensemble of raindrops. The model was used to examine the effect of a mean canting angle of both the propagation and backscatter medium, the standard deviation of the canting angles of the backscatter resolution volume, and polarization errors on the co-to-cross covariances and LDR. It was found that $\rho_{\mathrm{xH}}\left(\rho_{\mathrm{xV}}\right)$ is more sensitive to the mean backscatter canting angle than LDR. Furthermore, as the standard deviation of canting angle increases, LDR increases but $\rho_{\mathrm{xH}}\left(\rho_{\mathrm{xV}}\right)$ decreases. The $\rho_{\mathrm{xH}}\left(\rho_{\mathrm{xV}}\right)$ is also more sensitive to the mean propagation canting angle, $\theta$. As a practical application, examination of range profiles of $\rho_{\mathrm{xH}}\left(\rho_{\mathrm{xV}}\right)$ data along propagation paths with large $\phi_{\mathrm{dp}}$ may be a useful indicator of how well the "hybrid" method proposed for polarimetric upgrade of the WSR-88D would work (Doviak et al. 2000). The phases $\Psi_{\mathrm{xH}}\left(\Psi_{\mathrm{xV}}\right)$ are also very sensitive to the mean canting angles, $\alpha$ and $\theta$, as demonstrated in Fig. 6, especially after the accumulation of $30^{\circ}$ or $40^{\circ}$ of $\phi_{\mathrm{dp}}$. For these reasons, the co-to-cross covariances are particularly good for detecting small polarization errors. For CSU-CHILL data gathered during STEPS, it was noticed that whenever large accumulation of $\Psi_{\mathrm{dp}}$ was present, $\hat{\Psi}_{\mathrm{xH}}$ and $\hat{\Psi}_{\mathrm{xV}}$ would "saturate" around $90^{\circ}$ after the accumulation of $30^{\circ}$ to $40^{\circ}$ of $\phi_{\mathrm{dp}}$. If $\theta=0^{\circ}$ and no polarization errors were present, then the phases $\hat{\Psi}_{\mathrm{xH}}$ and $\hat{\Psi}_{\mathrm{xV}}$ would appear almost random in range profiles. Thus, because raindrops should not possess any preferred mean canting angle (other than zero), we believe this observed systematic phase behavior indicates the presence of small system polarization errors. Using the developed algorithm, the integrated errors were determined to be $\tau_{\mathrm{H}}=-0.5^{\circ}, \epsilon_{\mathrm{H}}=$ $-0.1^{\circ}, \tau_{\mathrm{V}}=89.5^{\circ}$, and $\epsilon_{\mathrm{V}}=0.4^{\circ}$. These polarization
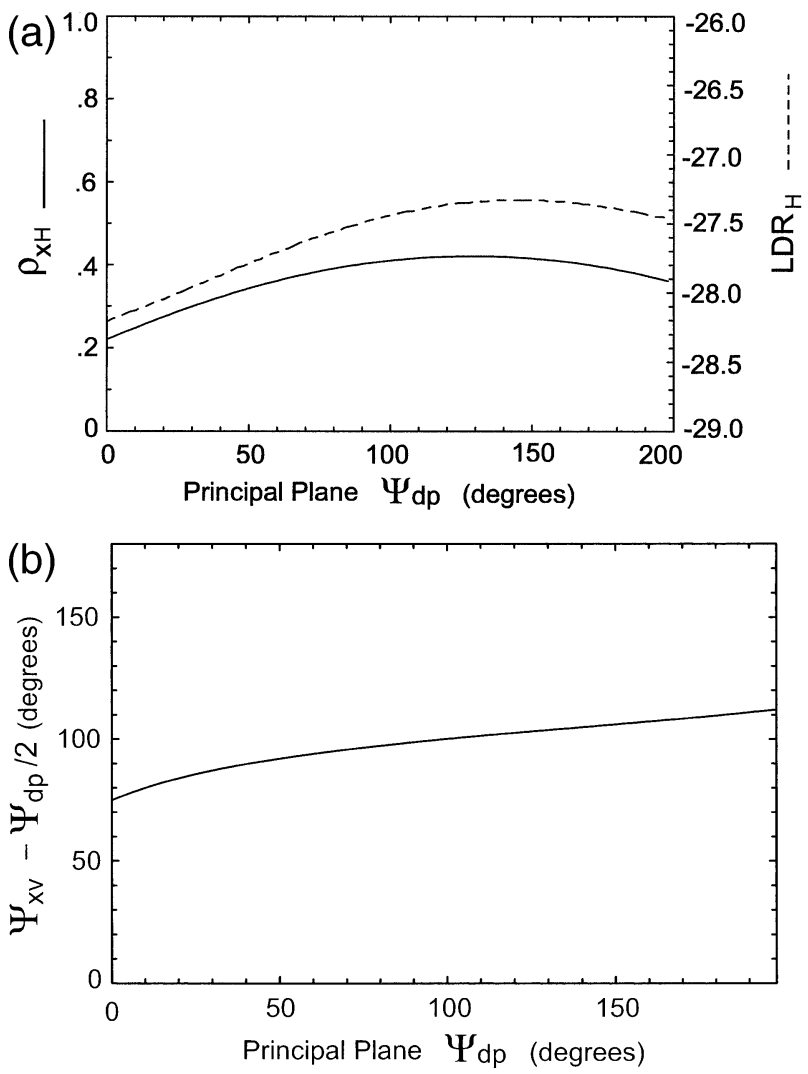

FIG. 15. Using the estimated polarization errors, (a) $\rho_{\mathrm{xH}}$ (solid), $\mathrm{LDR}_{\mathrm{H}}$ (dashed), and (b) $\Psi_{\mathrm{xH}}-\Psi_{\mathrm{dp}} / 2$ as functions of principal plane $\phi_{\mathrm{dp}}$ from the theoretical model.

errors were then used in the scattering model (with $\alpha$ $=\theta=0^{\circ}$ ) and the resulting $\rho_{\mathrm{xH}}, \mathrm{LDR}_{\mathrm{H}}$, and $\hat{\Psi}_{\mathrm{xH}}$ matched the behavior of the experimental range profiles very well, offering an explanation for the observed behavior of the data and lending credence to the overall methodology.

Thus, the co-to-cross covariances of weather radars signals potentially contain valuable information about not only precipitation microphysics, but also the calibration of the radar. By direct inspection of the co-tocross covariances in regions of large $\phi_{\mathrm{dp}}$ accumulation it can be seen if significant polarization errors are present or if a nonzero mean propagation canting angle exists. If polarization errors are present and can be well modeled by the $2 \times 2$ matrix representation given in this paper, then it is possible to remove these errors from the data. The application of the full covariance matrix data to microphysical retrieval will be given in a future paper.

Acknowledgments. The CSU-CHILL radar is operated by Colorado State University via a cooperative agreement with the National Science Foundation (NSF) ATM-9500108. The authors were supported by this grant and via NSF Grant ATM-9982030. The authors acknowledge the outstanding effort of Dave Brunkow, 
Pat Kennedy, and Robert Bowie in deploying and operating the radar for STEPS.

\section{REFERENCES}

Azzam, R. M. A., and N. M. Bashara, 1989: Ellipsometry and Polarized Light. North-Holland, 539 pp.

Bringi, V. N., and V. Chandrasekar, 2001: Polarimetric Doppler Weather Radar. Cambridge University Press, $636 \mathrm{pp}$.

- — N. Balakrishnan, and D. S. Zrnić, 1990: An examination of propagation effects in rainfall on radar measurements at microwave frequencies. J. Atmos. Oceanic Technol., 7, 829-840.

Brunkow, D., V. N. Bringi, P. C. Kennedy, S. A. Rutledge, V. Chandrasekar, E. A. Mueller, and R. K. Bowie, 2000: A discription of the CSU-CHILL National Radar Facility. J. Atmos. Oceanic Technol., 17, 1596-1608.

Caylor, I. J., and V. Chandrasekar, 1996: Time-varying ice crystal orientation in thunderstorms observed with multiparameter radar. IEEE Trans. Geosci. Remote Sens., 34, 847-858.

Chandrasekar, V., V. N. Bringi, and P. J. Brockwell, 1986: Statistical properties of dual-polarized signals. Preprints, $23 d$ Conf. on Radar Meteorology, Snowmass, CO, Amer. Meteor. Soc., 193-196.

Doviak, R. J., and D. S. Zrnić, 1993: Doppler Radar and Weather Observations. 2d ed. Academic Press, 562 pp.

—, V. N. Bringi, A. Ryzhkov, A. Zahrai, and D. S. Zrnić, 2000: Polarimetric upgrades to operational WSR-88D radars. J. Atmos. Oceanic Technol., 17, 257-278.

Hendry, A., Y. M. M. Antar, and G. C. McCormick, 1987: On the relationship between the degree of preferred orientation in precipitation and dual polarization radar echo characteristics. Radio Sci., 22, 37-50; Corrigendum, 22 (4), 687.

Holt, A. R., 1988: Extraction of differential propagation phase from data from S-band circularly polarized radars. Electron. Lett., $\mathbf{2 4}$ $1241-1242$

Huang, G. J., J. C. Hubbert, and V. Bringi, 2001: Precipitation canting angle distribution estimates from covariance matrix analysis of CSU-CHILL radar data. Preprints, 30th Int. Conf. on Radar Meteorology, Munich, Germany, Amer. Meteor. Soc., 651-653.

Hubbert, J. C., 1994: A comparision of radar, optic and specular null polarization theories. IEEE Trans. Geosci. Remote Sens., 32, 658-671.

_- and V. N. Bringi, 1995: An iterative filtering technique for the analysis of copolar differential phase and dual-frequency radar measurements. J. Atmos. Oceanic Technol., 12, 643-648.

—, and _ 1996: Specular null polarization theory: Applications to radar meteorology. IEEE Trans. Geosci. Remote Sens., 34, $859-873$.

- , and - 2001: Estimation of polarization errors from covariance matrices of CSU-CHILL radar data. Preprints, 30th Int. Conf. on Radar Meteorology, Munich, Germany, Amer. Meteor. Soc., 45-47.

,-- , and G. Huang, 1999: Construction and interpretation of
S-band covariance matrices. Preprints, 29th Int. Conf. on Radar Meteorology, Montreal, Quebec, Canada, Amer. Meteor. Soc., 205-207.

— - , and D. Brunkow, 2003: Studies of the polarimetric covariance matrix. Part I: Calibration methodology. J. Atmos. Oceanic Technol., 20, 696-706.

Kennaugh, E. M., 1949-1954: Research studies on the polarization properties of radar targets. Tech. Reps. 381-1 to 394-24, Electro Science Laboratory, Ohio State University.

Liu, L., V. N. Bringi, V. Chandrasekar, E. A. Mueller, and A. Mudukotore, 1994: Analysis of the copolar correlation coefficient between horizontal and vertical polarizations. J. Atmos. Oceanic Technol., 11, 950-963.

Lüneburg, E., V. Ziegler, A. Schroth, and K. Tragl, 1991: Polarimetric covariance matrix analysis for random radar targets. Proc. AGARD -Electromagnetic Wave Propagation Panel Symp. on Target and Clutter Scattering and Their Effects on Military Radar Performance, Ottawa, Ontario, Canada, NATO, AGARD, CP-501, 27-1-27-12.

Mardia, K. V., 1972: Statistics of Directional Data. Academic Press, $357 \mathrm{pp}$.

McCormick, G. C., 1981: Polarization errors in a two-channel system. Radio Sci., 16, 67-75.

Mott, H., 1992: Antennas for Radar and Communications: A Polarimetric Approach. John Wiley and Sons, $521 \mathrm{pp}$.

Mueller, E. A., 1984: Calculation procedures for differential propagation phase shift. Preprints, 22d Conf. on Radar Meteorology, Zurich, Switzerland, Amer. Meteor. Soc., 397-399.

Ryzhkov, A. V., 2001: Interpretation of polarimetric radar covariance matrix for meteorological scatterers: Theoretical analysis. J. Atmos. Oceanic Technol., 18, 315-328.

,-- J. C. Hubbert, V. N. Bringi, J. Vivekanandan, E. A. Brandes, 2002: Polarimetric radar observations and interpretation of co-cross-polar correlation coefficients. J. Atmos. Oceanic Technol., 19, 340-354.

Sachidananda, M., and D. S. Zrnić, 1986: Differential propagation phase shift and rainfall rate estimation. Radio Sci., 21, 235-247.

Sinclair, G., 1950: The transmission and reception of elliptically polarized waves. Proc. IRE, 38, 148-151.

Tragl, K., 1990: Polarimetric radar backscattering from reciprocal random targets. IEEE Trans. Geosci. Remote Sens., 8, 856-864.

—_ E. Lüneburg, A. Schroth, and V. Ziegler, 1991: A polarimetric covariance matrix concept for random radar targets. Proc. Seventh Int. Conf. on Antennas and Propagation, York, United Kingdom, Institution of Electrical Engineers and the International Union of Radio Science, Publ. 333, Series 7, 396-399.

van de Hulst, H. C., 1957: Light Scattering by Small Particles. Wiley, $970 \mathrm{pp}$.

Vivekanandan, J. V., W. M. Adams, and V. N. Bringi, 1991: Rigorous approach to polarimetric radar modeling of hydrometeor orientation distributions. J. Appl. Meteor., 30, 1053-1063.

Waterman, P. C., 1971: Symmetry, unitarity and geometry in electromagnetic scattering. Phys. Rev., D3, 825-839. 
Copyright $\odot 2003$ EBSCO Publishing 